\title{
Factores de riesgo que influyen en la disolución de las empresas del sector de fabricación de muebles de madera
}

\begin{abstract}
Risk factors that influence the dissolution of companies in the wooden furniture manufacturing sector
\end{abstract}

Salguero Barrionuevo Juan José. ${ }^{1}$ \& Padilla Martínez Mario Patricio. ${ }^{2}$

Abstract.

DOI: $\underline{\text { https://doi.org/10.33262/concienciadigital.v3i3.1.1453 }}$

This study deals with the risk factors that influence the dissolution of companies in the wood furniture manufacturing sector in the province of Tungurahua, this sector has become an economic alternative to traditional agriculture, poultry and livestock in the rural sector of the province. The main objective of the investigation is to determine the current situation of the sector in relation to the vulnerability of internal risk factors: administrative, financial, operational and strategic. The research methodology is descriptive, cross-sectional and correlational. In parallel, the research instrument was a questionnaire made up of 14 questions on the dimensions of administrative, financial, operational and strategic risks. The first 5 questions of the questionnaire are general, the The remaining 9 questions are built on a Likert scale: very low, low, medium, high and very high on the risk dimensions. The questionnaire was applied to a sample made up of 40 businessmen from the sector. The main results showed an acceptable instrument reliability by Cronbach's Alpha (0.744), most of the companies are located in the Pelileo canton (52.5\%), on average they have 10 employees who carry out the operational and commercial activities in the company, 5 years of operation on average and

${ }^{1}$ Universidad Técnica de Ambato, Facultad de Ciencias Administrativas, juanjokarts@gmail.com ${ }^{2}$ Universidad Técnica de Ambato, Facultad de Ciencias Administrativas, mp.padilla@uta.edu.ec 
according to the type of company most are small with $60 \%$, the most significant risk levels in the sector are: operational (39.00\%) and strategic (36.60\%) which influence the dissolution of companies.

Key words: Dissolution, factors, risks, wooden furniture.

\section{Resumen}

El presente estudio trata sobre los factores de riesgo que influyen en la disolución de las empresas del sector de fabricación de muebles de madera en la provincia de Tungurahua, este sector se ha convertido en una alternativa económica a la tradicional agricultura, avicultura y ganadería en el sector rural de la provincia. El principal objetivo de la investigación es determinar la situación actual del sector en relación a la vulnerabilidad de los factores de riesgo internos: administrativos, financieros, operativos y estratégicos. La metodología de la investigación es de tipo descriptiva, transversal y correlacional, paralelamente el instrumento de investigación fue un cuestionario conformado por 14 preguntas sobre las dimensiones de los riesgos administrativos, financieros, operativos y estratégicos, las primeras 5 preguntas del cuestionario son generales, las 9 preguntas restantes están construidos sobre una escala de Likert.: muy bajo, bajo, medio, alto y muy alto sobre las dimensiones de riesgo. El cuestionario fue aplicado a una muestra compuesta por 40 empresarios del sector. Los principales resultados mostraron una confiabilidad aceptable de instrumento por Alfa de Cronbach (0.744), la mayor parte de las empresas se ubican en el cantón Pelileo (52.5\%), en promedio tienen 10 empleados que realizan las actividades operativas y comerciales en la empresa, 5 años de funcionamiento en promedio y según el tipo de empresa la mayor parte son pequeñas con el 60\%, los niveles de riesgos más significativo en el sector son: operativos (39.00\%) y estratégico (36.60\%) los cuales influyen en la disolución de las empresas.

Palabras claves: Disolución, factores, riesgos, muebles de madera.

\section{Introducción.}

La fabricación de muebles de madera es uno de los sectores económicos más importantes en la provincia de Tungurahua, en los últimos 20 años ha permitido dinamizar la economía principalmente del sector rural. Como principal factor que ha impulsado al sector es la necesidad de buscar alternativas a los sistemas de producción tradicional debido a los efectos 
negativos que produjo la erupción del volcán Tungurahua, por lo tanto se realizó el cambio de la matriz productiva de la agricultura, avicultura y ganadería hacia la producción artesanal de muebles de madera en gran parte de la provincia.

Los principales puntos de producción y comercialización de muebles de madera en la provincia de Tungurahua son: Ambato, Pelileo y Píllaro, especialmente la parroquia rural de Huambaló ubicada a 7 kilómetros de la ciudad de Pelileo, es la más conocida a nivel regional y nacional, actualmente el tipo producción es artesanal y familiar. Uno de los factores más importantes que ha fomentado el desarrollo de este sector es la educación, capacitación y tecnificación del sector fomentado por el Centro Artesanal Huambaló "CENARHU” la primera organización establecida jurídicamente en la parroquia por artesanos ebanistas (Veloz, 2015). Actualmente Huambaló cuenta 40 talleres artesanales dedicados a la fabricación de muebles de madera y 80 locales comerciales, además emplea alrededor de 921 personas (Gobierno Autónomo Descentralizado Parroquial Rural Huambaló, 2015).

El sector ha experimentado un crecimiento económico exponencial en la última década, ha facturado de 1,5 millones a 4 millones de dólares anuales en los últimos años, los meses en los cuales se tiene un mayor promedio de ventas es diciembre gracias a los feriados navideños y de fin de año, el principal mercado es Quito al cual se destina que el 80 \% de la producción local (Barreno, 2015). Sin embargo, como ocurre en todos los sectores, los riesgos debilitan y son en parte causantes de la disolución de las empresas. Si se analiza el contexto externo del sector los principales factores de riesgo son: vías de segundo y tercer orden se encuentran en mal estado lo que dificulta el transporte, la escasa accesibilidad a la tecnología tanto de comunicaciones como productiva, descoordinación institucional de los actores territoriales, escaso apoyo de los entes públicos reguladores, amenazas naturales por eventos sísmicos o volcánicos, escasas fuentes de financiación (Gobierno Autónomo Descentralizado Parroquial Rural Huambaló, 2015).

Estudios anteriores demuestran que actualmente se han empezado a manejar diferentes concepciones y términos aplicados sobre la gestión de riesgos, algunos sobre una perspectiva tradicional de prevenir y otros en la búsqueda de planteamientos innovadores de estrategia para reducción de vulnerabilidades, la mitigación y aprovechar las oportunidades que también traen los riesgos (Martínez \& Blanco, 2017). 
Los factores internos que afectan a las empresas del sector de fabricación de muebles se pueden agrupar en los siguientes segmentos: riesgo de crédito, legal, de mercado, operacional, estratégico, liquidez y riesgo ambiental (Klann, 2014). Por lo tanto, los riesgos que afectan a las empresas provienen de fuentes internas como externos. El presente estudio determina como los factores de riesgos influyen en la disolución de las empresas del sector de fabricación de muebles de madera de la provincia de Tungurahua para lo cual, se realizó una investigación descriptiva, transversal y correlacional basada en el estudio teórico y empírico, la recopilación de datos se los realizo mediante un cuestionario aplicado a una muestra de 40 empresas, que fue validado por el índice de confiabilidad Alfa de Cronbach,, la determinación del nivel de riesgo se determinan bajo la escala de Likert: muy bajo, bajo, medio, alto y muy alto, el análisis estadístico inferencial se realizó por Correlación de Pearson.

\section{Estado de Arte.}

\section{Factores de riesgos}

Los factores de riesgo son inherentes de forma permanente a las actividades empresariales y con el pasar del tiempo se han vuelto más complejos que obligan a las empresas a adaptarse, ajustarse y prepararse ante los cambios en un entorno tan volátil (Martínez \& Blanco, 2017). Los principales cambios tuvieron una mayor notoriedad en el ámbito empresarial en la época de los 90 con el desarrollo del neoliberalismo económico caracterizado por la incertidumbre en los mercados principalmente el financiero, la crisis económica mundial, los escándalos financieros fueron los principales condiciones que marcaban un nuevo entorno económico mundial (Andreeva \& Ritala, 2016). Estos cambios trajeron una mayor influencia sobre la pérdida de confianza en los mercados de valores lo que evidencio la necesidad y urgencia de gestionar mecanismos y estrategias para restaurar la credibilidad en los mercados.

Así es como nace la Gestión de Riesgos un enfoque gerencial que se centra en analizar, evaluar, prevenir o dar soluciones para evitar o minimizar los efectos de los riesgos a los cuales las empresas se enfrentan diariamente (Castillo, 2017). El riesgo empresarial se puede definir como cualquier incertidumbre que afecta la consecución de los objetivos de una organización, por lo tanto, influyen en la supervivencia y desarrollo de la empresa, 
dependiendo el grado de preparación de una empresa el riesgo puede representar una amenaza que afecte la continuidad de una empresa o ser transformada en una oportunidad mediante acciones estratégicas inteligentes que permitan convertir amenazas en oportunidades (Jaraba, Nuñez, \& Villanueva, 2018).

Una forma generalizada y amplia de clasificar a los riesgos es mediante la determinación de grupos, los riesgos se pueden clasificar en riesgos: operativos los cuales son derivados de las actividades y procesos utilizados en la producción de bienes o servicios, los financieros surgen de la inestabilidad de los mercados y la economía real, los de cumplimiento son aquellos relacionados a las normativas, políticas y legislaciones y los riesgos estratégicos son aquellos enfocados a la competitividad, innovación, el mercado, los competidores y la industria en general (Morón, Reyes \& Antonio 2015).

Los riesgos estratégicos representan el impacto para la empresa generado por los cambios en el nivel económico, social y político que rodean a la empresa (Castillo, Gamboa, \& Castillo, 2019). También puede entenderse como el resultado de una mala gestión empresarial en relación con la competencia, la innovación y el desarrollo (Oliveira, 2016). Los riesgos de tipo operativo son las pérdidas en relación con el mal desempeño de las funciones como resultado de fallas o deficiencias en procesos internos, personas y sistemas dentro de las empresas (Zonatto \& Beuren, 2010). Los riesgos financieros son derivados del resultado de cambios en variables financieras como la tasa de interés y la tasa de cambio, o el resultado de posibles pérdidas en los mercados financieros, el riesgo refleja la exposición de la empresa a las operaciones financieras, como resultado de una gestión ineficaz con respecto a los flujos de efectivo (Oliveira, 2016). El riesgo legal es el resultante de los cambios en los requisitos legales y reglamentarios que la organización no tiene en cuenta o no cumple, por lo tanto la empresa se expone a multas o sanciones, por lo tanto, existe la posibilidad de un impacto negativo en la empresa (Zonatto \& Beuren, 2010).

Los tipos de riesgos a los cuales se enfrenta una empresa dependen del tipo de actividades que desarrolla, las características propias de la industria y del mercado en que se desenvuelve, es decir tanto estos riesgos dependen del entorno interno y externo en el que se desenvuelve una organización (Capa, Capa, \& Ollague, 2018). De esta forma en el sector de fabricación de muebles de madera se deben identificar los factores que generan riesgos y crear una 
jerarquía de riesgos a través de la determinación de niveles de riesgos: alto, medio y bajo, con esto se debe establecer prioridades de atención sobre los cuales se implementarán los mecanismos que contribuyan a la mitigación o eliminación de los niveles de riesgos.

Es importante considerar la evaluación de la probabilidad de ocurrencia de un riesgo para lo cual la probabilidad se evalúa de manera cualitativa considerando escala: bajo, medio y alto, de forma que se reconozca la evaluación del impacto. Para ello Morón, Reyes, y Antonio (2015) proponen la determinación de los niveles de riesgo ver tabla 1 en base a la siguiente escala: bajo en un rango hasta el $50 \%$, medio entre $51 \%$ al $75 \%$ y alto desde $76 \%$ hasta $100 \%$, para lo cual se considera la calificación total obtenida de la matriz de riesgos multiplicado por 100 y dividido por la ponderación total. La matriz de riesgos es una de las herramientas más valiosas para evaluar el potencial de los riesgos, este instrumento permite dinamizar de forma cualitativa y cuantitativa a partir del análisis de probabilidad e impacto en base a parámetros previamente asignados (Gutiérrez \& Sánchez, 2018).

Tabla 1 Niveles de riesgos

\begin{tabular}{ll}
\hline Nivel de riesgo & Rango \\
\hline Alto & $76 \%-100 \%$ \\
Mediano & $51 \%-75 \%$ \\
Bajo & $0 \%-50 \%$ \\
\hline
\end{tabular}

Fuente: Morón, Reyes, \& Antonio (2015)

El sector de fabricación de muebles de madera al igual que todas las industrias está atravesando por una situación de mayor competitividad causado por los efectos de la globalización, la apertura comercial, el avance de la tecnología, las facilidades para la logística y problemas ambientales que exige cambios y respuestas eficientes a las empresas para que puedan sobrevivir y crecer en un contexto de continuo cambio, en el cual el mercado define el éxito o fracaso (Serna, 2016).

\section{Industria de fabricación de muebles de madera en el Ecuador}

La industria de la fabricación de muebles de madera en el Ecuador está dentro de un segmento más amplio denominada industria maderera, del cual dependen para la obtención de materias primas, esta industria está compuesta de la siguiente manera grafico 1 (CORPEI, 2012): 


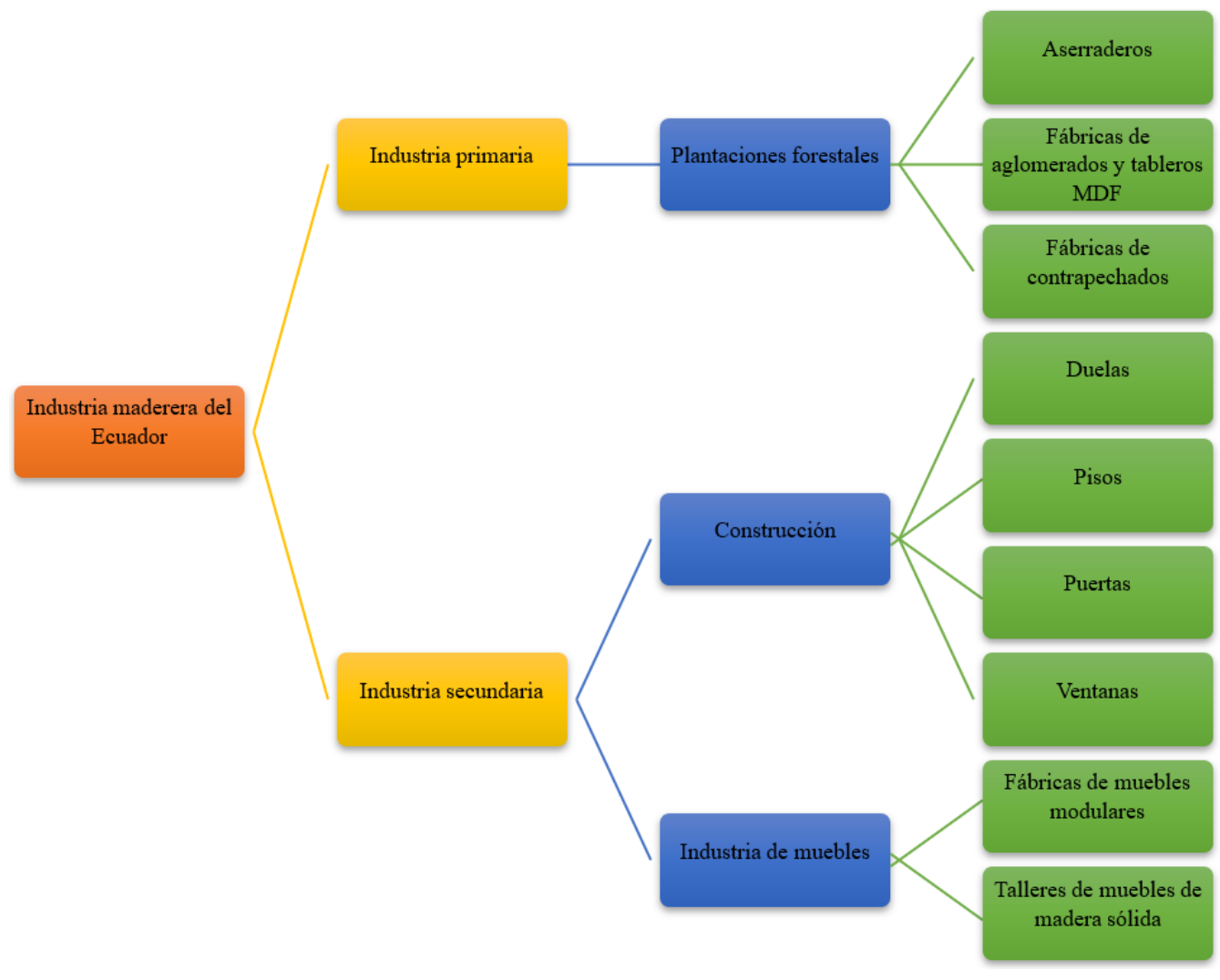

Gráfico 1 Industria maderera en el Ecuador Adaptado de CORPEI, 2012

La industria secundaria de fabricación de muebles tiene dos grandes segmentos diferenciados ver tabla2, entre las que se destacan las grandes fábricas de construcción de muebles modulares y los talleres de muebles de madera sólida, la presente investigación se centra en los talleres y pequeñas fábricas de producción de muebles de madera.

Tabla 2 Características de la industria de muebles de madera en el Ecuador

\begin{tabular}{ll}
\hline $\begin{array}{l}\text { Elementos de la } \\
\text { industria secundaria } \\
\text { de muebles }\end{array}$ & Características \\
\hline $\begin{array}{l}\text { Fábricas de muebles } \\
\text { modulares }\end{array}$ & $\begin{array}{l}\text { Son aquellas fábricas de muebles que utilizan materiales como tableros, MDF, } \\
\text { generalmente proveen de muebles de oficina, divisiones, escritorios, mesas de } \\
\text { conferencia y sillas, gabinetes de baño y de cocina a grandes empresas en el } \\
\text { Ecuador. } \\
\text { Este sector se caracteriza porque tiene pocos problemas relacionados a la } \\
\text { producción y fabricación ya que manejan plantas con tecnología de punta. }\end{array}$ \\
$\begin{array}{l}\text { Talleres y pequeñas } \\
\text { fábricas de muebles }\end{array}$ & $\begin{array}{l}\text { Este sector tiene muchos problemas debido a que los procesos, las herramientas y } \\
\text { maquinaria son generalmente artesanales y con escasa implementación de } \\
\text { tecnología, lo cual impide la industrialización de este segmento. }\end{array}$
\end{tabular}

Adaptado de CORPEI, 2012 
Otro de los grandes problemas producidos en los talleres y pequeñas fábricas de muebles de madera es que la mayor parte de madera aserrada que consumen proviene de madera producida con motosierra, lo cual genera un alto porcentaje de desperdicios y madera mal escuadrada y de superficie irregular (Peralta, 2009).

\section{Disolución de empresas}

La disolución es la primera etapa para consolidar la desaparición legal de una organización, esta etapa consiste en que los socios acuerdan realizar la forma y los trámites necesarios para la liquidación y extinción de una organización (Nieto, 2004). En este sentido la disolución se da cuando los socios han determinado la inviabilidad de continuar con la existencia de una organización principalmente debido a motivos financieros, baja posición o aceptación en el mercado.

En el contexto ecuatoriano las nuevas empresas no superan la barrera de los tres meses debido a la incertidumbre del contexto empresarial endógeno y exógeno (Maiza, Rivera, \& Morales, 2020). El fracaso empresarial se da generalmente cuando la empresa está inmersa en un estado crítico debido a la falta de experiencia, preparación y capacitación de los administradores de las empresas al momento de tomar decisiones financieras, estratégicas y de inversión.

La disolución de una organización puede ser intencionado para evitar pérdidas futuras, ya que el propietario o los socios han detecto a tiempo que la organización ya no genera los rendimientos financieros esperados lo que hace que la empresa deje de ser competitiva tomando la decisión de liquidar o vender la empresa.

\section{Metodología.}

La presente investigación es de tipo descriptivo, transversal y correlacional, debido a que se basa en la determinación de los principales factores de riesgos que atraviesan las empresas de fabricación de muebles de madera en la provincia de Tungurahua. El año en el que se realizó el presente estudio fue a finales del 2019 e inicios del 2020, el instrumento de investigación utilizado fue un cuestionario compuesto de 14 preguntas y validado por Alfa 
de Cronbach, fue aplicado a 40 gerentes de las empresas de fabricación de muebles de madera en la provincia de Tungurahua.

Las primeras 5 preguntas del cuestionario son generales, las 9 preguntas restantes están construidos sobre una escala de Likert en base a las dimensiones de riesgo administrativas, financieras, operativas y estratégicas. Para la determinación del nivel de riesgo se utilizó la escala de Likert: muy bajo, bajo, medio, alto y muy alto.

Para el análisis correlacional se utilizó Pearson, según Restrepo \& González (2007) la correlación permite determinar el grado de las relaciones entre variables cuantitativas en un rango de -1 a 1 . El objetivo del presente estudio es determinar los principales factores de riesgo y su relación con la disolución de las empresas de fabricación de muebles de madera en la provincia de Tungurahua.

\section{Resultados}

Tabla 3 Resultados perfil de las empresas

\begin{tabular}{ll}
\hline Variables & Resultados \\
\hline Instrucción del empresario & Primaria 7.5\% \\
& Secundaria 42.5\% \\
& Pregrado 47.5\% \\
& Posgrado 2.5\% \\
Sector en donde se ubica la empresa & Pelileo 52.5\% \\
& Ambato 32.5\% \\
& Tisaleo 15.0\% \\
Número de empleados & 10 empleados promedio \\
Años de funcionamiento & 5 años de funcionamiento en promedio \\
Tipo de empresas & Micro 40\% \\
& Pequeña 60\% \\
\hline
\end{tabular}

Fuente: Encuesta

De acuerdo al perfil de las empresas de fabricación de muebles de madera en la provincia de Tungurahua los principales resultados evidenciaron que la mayor parte de los empresarios tienen un nivel de instrucción promedio: pregrado $47.5 \%$, y secundaria $42.5 \%$. La mayor parte con el 52.5\% de las empresas se ubican en el cantón Pelileo, tienen en promedio 10 empleados que realizan las actividades operativas y comerciales en la empresa, tiene 5 años de funcionamiento en promedio y según el tipo de empresa la mayor parte son pequeñas con el $60 \%$. 


\section{Análisis inferencial}

\section{Alfa de Cronbach}

El coeficiente de Cronbach es una medida que expresa la confiabilidad de un instrumento en una escala de 0 a 1 , en donde 0 representa una nula confiabilidad y 1 máxima confiabilidad, se debe considerar para un estudio de investigación un valor entre 0.7 a 0.9 de confiabilidad (Cascaes da Silva, 2015).

Tabla 4 Alfa de Cronbach

\begin{tabular}{|c|c|}
\hline \multicolumn{2}{|c|}{ Estadísticas de fiabilidad } \\
\hline Alfa de Cronbach & $\mathrm{N}$ de elementos \\
\hline 0 & \\
\hline
\end{tabular}

Fuente: Encuesta

Con un valor 0.774 el instrumento de investigación de confiabilidad adecuado, se debe mencionar que las 9 preguntas corresponden a las dimensiones de riesgos administrativos, financieros, operativos y estratégicos los cuales están construidos con una escala de Likert.

\section{Correlación de Pearson}

Es una estadística inferencial que refleja la intensidad de la asociación lineal entre dos o más variables cuantitativas con un rango de -1 correlación perfecta negativa a +1 correlación perfecta positiva, se debe considerar un coeficiente mayor de 0.75 (Manterola, 2018). Mediante el coeficiente de Correlación de Pearson se determina el grado de correlación de los riesgos y su impacto al objeto de estudio.

Tabla 5 Correlaciones

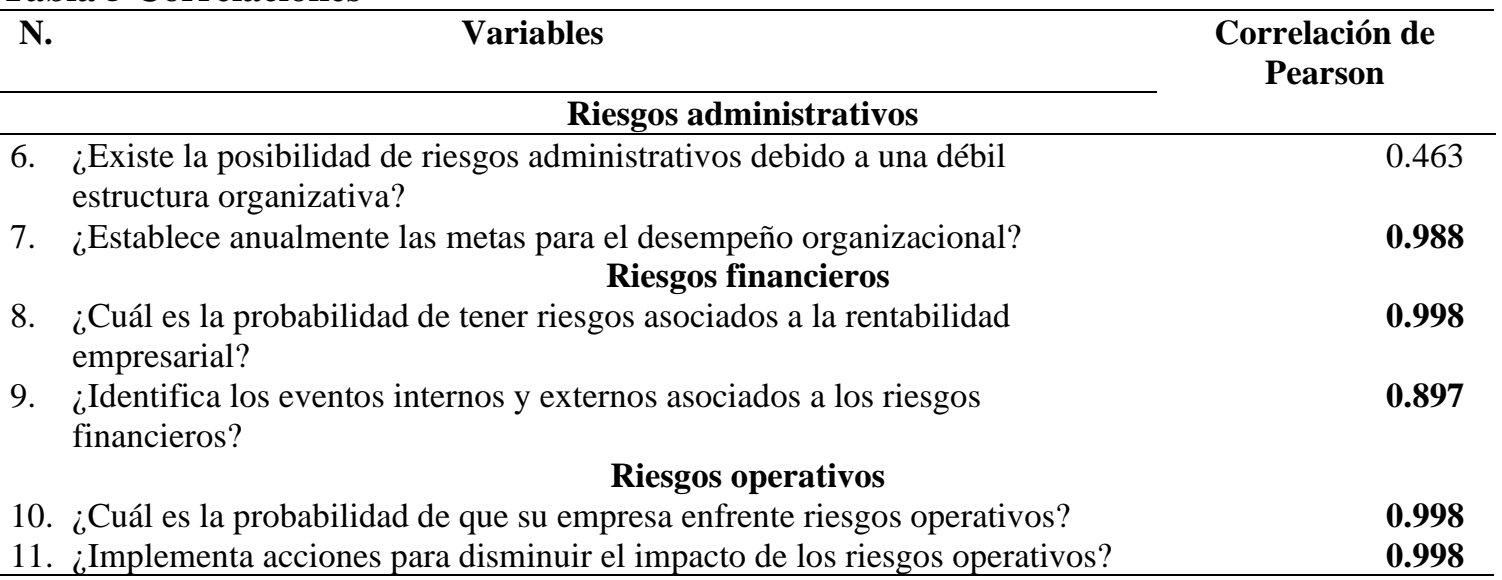


12. ¿Cuál es la probabilidad de que su empresa enfrente riesgos estratégicos debido al entorno externo cambiante?

13. ¿Evalúa y diseña estrategias para mitigar los efectos de los cambios

0.764 políticos, económicos y tecnológicos?

14. ¿Cuál es la probabilidad de que su empresa sea disuelta por problemas empresariales?

Fuente: Encuesta

La mayor parte de las variables con un índice de Correlación de Pearson tienen un coeficiente mayor a 0.75 , a excepción de las preguntas 6,12 y 13 . Por lo tanto se determina que estas variables tienen una correlación débil en relación a los riesgos que enfrentan las empresas fabricación de muebles de madera en la provincia de Tungurahua.

Tabla 6 Tipos de riesgos

\begin{tabular}{ll}
\hline Tipos de riesgos & Nivel de riesgos \\
\hline Administrativos & Bajo 24.4\% \\
& Medio 22.0\% \\
& Alto 39.0\% \\
& Muy alto 14.6\% \\
Financieros & Muy bajo 7.3\% \\
& Bajo 14.6\% \\
& Medio 29.3\% \\
& Alto 22.0\% \\
& Muy alto 26.8\% \\
Operativos & Bajo 24.4\% \\
& Medio 22.0\% \\
& Alto 39.0\% \\
& Muy alto $14.6 \%$ \\
Estratégicos & Bajo $19.5 \%$ \\
& Medio 7.3\% \\
& Alto 36.6\% \\
& Muy alto 36.5\% \\
\hline
\end{tabular}

Fuente: Encuesta

Alto 36.6\%

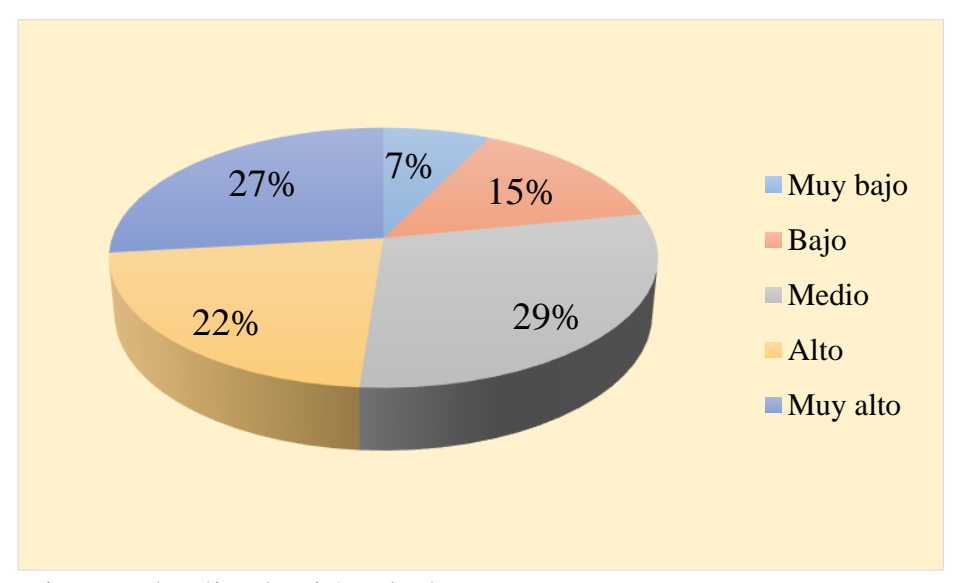

Gráfico 2 Riesgo de disolución de la empresa

Fuente: Encuesta 
De acuerdo a los empresarios consultados, la probabilidad de riesgo administrativo en las fábricas de muebles de madera de la provincia de Tungurahua es alto con el $39.0 \%$, en relación a los riesgos financieros es medio con el 29.3\%, en los riesgos de tipo operativo los riesgos son altos con el 39.0\% y en el riesgo de tipo estratégico es muy alto con el 36.5\%. El riesgo de disolución de la empresa de fabricación de muebles de madera en la provincia de Tungurahua es medio con el $29.30 \%$.

\section{Discusión.}

Sobre los 4 tipos de riesgos que las empresas de producción de muebles de madera deben enfrentar los más representativos son los riesgos administrativos y operativos con mayor probabilidad riesgos de nivel alto (39.0\%) y de tipo estratégicos con probabilidad de nivel alto con $36.6 \%$. En relación a los riesgos administrativos estos están relacionados a la debilidad de establecer una estructura organizativa definida, y la omisión del establecimiento de metas.

En relación a los riesgos operativos estos fueron determinados en relación de las probabilidades y acciones para disminuir el impacto sobre las actividades y procedimientos dentro de la industria como: obtención de materias primas, corte, diseño, armado y tapizado debido a que la sector maneja procesos poco industrializados o artesanales. Con respecto al riesgo de disolución de las empresas este tiene un impacto medio con el $29.30 \%$, por lo cual se considera que los empresarios toman decisiones para evitar el cierre de empresas y pérdidas de puestos de empleo.

El presente estudio sirve de base para mejorar las decisiones dentro del sector, fomentar la atención a los riesgos internos: administrativos, financieros, operativos y estratégicos los cuales son inherentes a la industria y el mercado, con el fin de elaborar estrategias que permitan disminuir o mitigar el impacto negativo que trae consigo los riesgos.

\section{Conclusiones.}

- La industria de la fabricación de muebles de madera en la provincia de Tungurahua se ha caracterizado por ser un sector alternativo a la matriz productiva tradicional relacionada a la agricultura, avicultura o ganadería, por lo cual es importante su 
desarrollo y crecimiento como aporte a la generación de fuentes de empleo locales e ingresos.

- Los riesgos son inherentes a las actividades empresariales, de acuerdo a la literatura empresarial revisada, las investigaciones señalan que es importante evaluar y tomar medidas correctivas que permitan mitigar o minimizar el efecto de los riesgos sobre las actividades empresariales.

- El perfil general de una empresa de fabricación de muebles de madera es el siguiente: el empresario tiene estudios de pregrado (47.5\%), el sector en donde mayoritariamente se ubican en el cantón Pelileo (52.5\%), tiene en promedio 10 empleados, 5 años de funcionamiento en promedio y es de tipo pequeña empresa $(60 \%)$.

- Los principales riesgos a los cuales se ven enfrentado las empresas de fabricación de muebles de madera en la provincia de Tungurahua se clasifican de tipo administrativo el mayor resultado es alto (39.0\%), en relación a los riesgos financieros el riesgo es medio (29.3\%), en los riesgos de tipo operativo los riesgos son altos (39.0\%) y en el riesgo de tipo estratégico es muy alto (36.5\%). El riesgo de disolución de la empresa de fabricación de muebles de madera en la provincia de Tungurahua es medio $(29.30 \%)$.

\section{Referencias Bibliográficas}

Andreeva, T., \& Ritala, P. (2016). What are the sources of capability dynamism? Reconceptualizing dynamic capabilities from the perspective of organizational change. Baltic Journal of Management, 238-259.

Barreno, V. P. (2015). La asociatividad y su incidencia en los ingresos de los productores de los muebles de la parroquia Huambaló del cantón Pelileo. Ambato: Universidad Técnica de Ambato.

Capa, L., Capa, X., \& Ollague, J. (2018). Estructura de capital en las pequeñas y medianas empresas bananeras de la provincia de El Oro. Revista Universidad y Sociedad, 294303. Obtenido de http://scielo.sld.cu/pdf/rus/v10n2/2218-3620-rus-10-02-304.pdf

Cascaes da Silva, F. (2015). Estimadores de consistencia interna en las investigaciones en salud: el uso del coeficiente alfa. Revista Peruana de Medicina Experimental y Salud Publica. 
Castillo, C. (2017). Sistema de administración de operaciones para el sector frutícola del cantón Cevallos provincia de Tungurahua. Ambato: Universidad Técnica de Ambato. Obtenido de https://repositorio.uta.edu.ec/bitstream/123456789/25764/1/365\%20o.e..pdf

Castillo, C., Gamboa, J., \& Castillo, D. (2019). Aproximación del modelo CAGE para la internacionalización de los productos textiles del Ecuador. Estudios de la Gestión: revista internacional de administración, 9-35. Obtenido de https://revistas.uasb.edu.ec/index.php/eg/article/view/1192/1104

CORPEI. (2012). Planeación Estratégica 2007 - 2012. Quito.

Gobierno Autónomo Descentralizado Parroquial Rural Huambaló. (2015). Plan de Desarrollo y Ordenamiento Territorial de la parroquia rural Huambaló. Centro de Ingeniería y GeoInformación Ambiental .

Gutiérrez, Y., \& Sánchez, A. (2018). Diseño de un Modelo de Gestión de Riesgos basado en ISO 31.000:2012 para los Procesos de Docencia de Pregrado en una Universidad Chilena. Formación universitaria, 15-32. Obtenido de https://scielo.conicyt.cl/pdf/formuniv/v11n4/0718-5006-formuniv-11-04-00015.pdf

Jaraba, I., Nuñez, M. A., \& Villanueva, E. (2018). Riesgos estratégicos. Un estudio de las medidas de tratamiento implementadaspor las grandes empresas privadas de Antioquia, Colombia. Cuadernos de Contabilidad, 171-181. Obtenido de http://www.scielo.org.co/pdf/cuco/v19n47/0123-1472-cuco-19-47-00171.pdf

Klann, R. (2014). Fatores de Risco Evidenciados Pelas Maiores Empresas Listadas na Bm\&Fbovespa. Revista de Gestão Ambiental e Sustentabilidade.

Maiza, C., Rivera, P., \& Morales, D. (2020). El fracaso de la actividad emprendedora en el contexto latinoamericano. Uniandes EPISTEME, 162-176. Obtenido de http://45.238.216.13/ojs/index.php/EPISTEME/article/view/1490/1099

Manterola, C. (2018). Confiabilidad, precisión o reproducibilidad de las mediciones. Métodos de valoración, utilidad y aplicaciones en la práctica clínica. Laboratorio e Infectología, 680-688.

Martínez, R., \& Blanco, M. (2017). Gestión de riesgos: reflexiones desde un enfoque de gestión empresarial emergente. Revist a Venezolana de Gerencia. Obtenido de https://www.redalyc.org/jatsRepo/290/29055967009/html/index.html

Morón, A., Reyes, M., \& Antonio, Á. (2015). Gestión de riesgos en la empresa R.C. Agelvis, C.A. MULTICIENCIAS, 417-427.

Nieto, A. (2004). Profesores de Enseñanza Secundaria. Administracion de Empresas. Volumen I. Sevilla: Editorial MAD. 
Oliveira, F. P. (2016). Gestão do Risco nas PME de Excelência Portuguesas. Tourism \& Management Studies.

Peralta, N. (2009). La industria maderera nacional, incidencia tributaria en su proceso productivo y de comercialización hasta el año 2009. Quito: Universidad Andina Simón Bolívar Sede Ecuador.

Restrepo, L., \& González, J. (2007). Revista Colombiana de Ciencias Pecuarias. De Pearson a Spearman.

Serna, A. L. (2016). Caracterización de la productividad de las ebanisterías de Quibdó, Chocó-Colombia. Entramado, 206-219.

Veloz, J. (2015). Dinámicas de desarrollo rural e inclusión social en la parroquia Huambaló en Tungurahua. Quito: Facultad Latinoamericana de Ciencias Sociales. Obtenido de http://repositorio.flacsoandes.edu.ec:8080/bitstream/10469/8564/2/TFLACSO2015JNVS.pdf

Zonatto, \& Beuren. (2010). Categorias de Riscos Evidenciadas nos Relatórios da Administração de Empresas Brasileiras com ADRs. Revista Brasileira de Gestão de Negócios, 41-155. 


\section{PARA CITAR EL ARTÍCULO INDEXADO.}

Salguero Barrionuevo, J. J., \& Padilla Martínez, M. P. (2020). Factores de riesgo que influyen en la disolución de las empresas del sector de fabricación de muebles de madera. ConcienciaDigital, 3(3.1), 383-398. https://doi.org/10.33262/concienciadigital.v3i3.1.1453

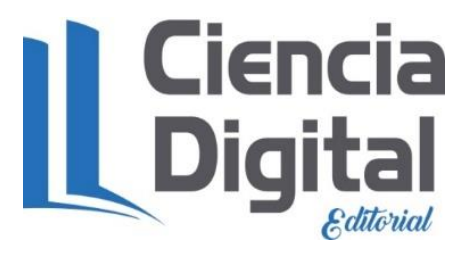

El artículo que se publica es de exclusiva responsabilidad de los autores y no necesariamente reflejan el pensamiento de la Revista Conciencia Digital.

El artículo queda en propiedad de la revista y, por tanto, su publicación parcial y/o total en otro medio tiene que ser autorizado por el director de la Revista Conciencia Digital.
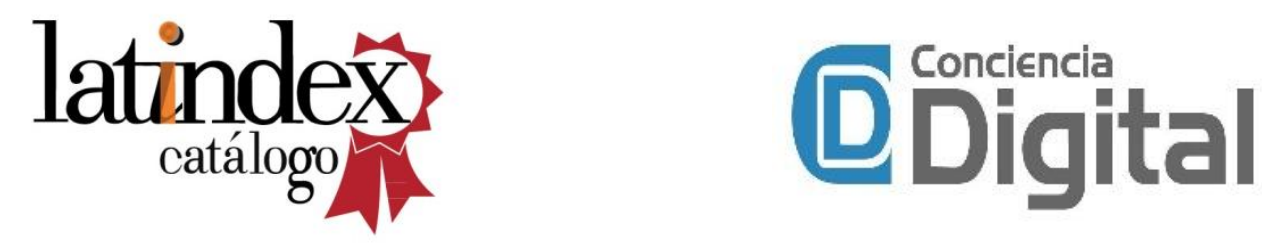

\section{Servicio}

